\title{
Magnetic resonance/ultrasound fusion targeted biopsy of the prostate can be improved by adding systematic biopsy
}

\author{
Victor Cauni ${ }^{1}$, Dan Stanescu ${ }^{2}$, Florin Tanase', Bogdan Mihai ${ }^{1}$, Cristian Persu ${ }^{3}$ \\ ${ }^{1}$ Urology Department, Colentina Clinical Hospital, ${ }^{2}$ Nuclear Medicine Department, Fundeni Clinical Institute, ${ }^{3 “}$ Carol \\ Davila" University of Medicine and Pharmacy, Department of Urology, Bucharest, Romania
}

\begin{abstract}
Aim: Magnetic resonance/ ultrasound fusion targeted biopsy (Tbs) is widely used for diagnosing prostate cancer (PCa). The aim of our study was to compare the cancer detection rate (CDR) and the clinically significant prostate cancer detection rate (csPCa) of the magnetic resonance/ultrasound fusion targeted biopsy with those of the standard systematic biopsy (Sbs) and of the combination of both techniques. Material and methods: A total of 182 patients underwent magnetic resonance/ ultrasound fusion Tbs on the prostate for PCa suspicion based on multiparametric magnetic resonance imaging (mMRI) detection of lesions with PI-RADSv2 score $\geq 3$. A total of 78 patients had prior negative biopsies. Tb was performed by taking 2-4 cores from each suspected lesion, followed by Sb with 12 cores. We evaluated the overall detection rate of PCa and clinically significant prostate cancer, defined as any PCa with Gleason score $\geq 3+4$. Results: Median prostate specific antigen (PSA) level pre-biopsy was $7.4 \mathrm{ng} / \mathrm{ml}$ and median free-PSA/PSA ratio was $10.2 \%$. Patient median age was 62 years old. PIRADSv2 score was 3 in 54 cases, 4 in 96 cases and 5 in 32 cases. PI-RADS-dependent detection rate of Tbs for scores 3, 4 and 5 was $25.9 \%, 65.6 \%$ and $84.4 \%$, respectively, with csPCa detection rates of $24.1 \%, 54.2 \%$, and $71.9 \%$. Overall detection rate was $57.1 \%$ for Tbs, which increased to $60.4 \%$ by adding Sbs results. Detection rate for clinically significant prostate cancer (csPCa) was $48.4 \%$ and increased to $51.1 \%$ by adding Sbs. Overall detection rate for repeated biopsy was $50 \%$ and $68.3 \%$ for biopsy in naïve patients. Sbs detection rate was $55.5 \%, 8$ patients having a negative biopsy on Tbs. Conclusions: When Tbs is considered due to a PI-RADS $\geq 3$ lesion on mMRI, combined Tbs + Sbs increases the overall CDR and csPCa detection rates.
\end{abstract}

Keywords: mMRI; fusion biopsy; targeted biopsy; systematic biopsy; prostate cancer

\section{Introduction}

The topic of targeted biopsy for prostate cancer diagnosis is of great interest considering the advances in medical imaging, software development and the high frequency of this condition in the aging male. Multiparametric magnetic resonance imaging (mMRI) is being indicated prior to prostate biopsy and many studies have

Received 12.11.2021 Accepted 15.03.2021

Med Ultrason

2021, Vol. 23, No 3, 277-282

Corresponding author: Cristian Persu

Urology Department, Colentina Clinical

Hospital, Bucharest, Romania

E-mail: drcpersu@gmail.com

Phone: +40722302225 shown the superiority of targeted biopsy (Tbs) compared to systematic biopsy (Sbs) alone [1-7]. The current European Association of Urology (EAU) guidelines on prostate cancer strongly recommend performing mMRI in all patients with a suspicion of prostate cancer (PCa) [8]. If mMRI shows a lesion classified by a Prostate Imaging Reporting and Data System version 2 (PI-RADSv2) [9] score of 3 or more, magnetic resonance/ultrasound fusion Tbs should be performed.

Tbs alone remains controversial and guidelines still recommend combining Tbs with Sbs especially in patients with prior negative biopsies. EAU guidelines give a weak recommendation for Tbs alone in the rebiopsy setting [8]. Also, it is unclear if adding Sbs to Tbs leads to a higher detection rate of nonsignificant $\mathrm{PCa}$. The value of combined Tbs and Sbs, especially after prior negative biopsy series, needs to be clarified. 


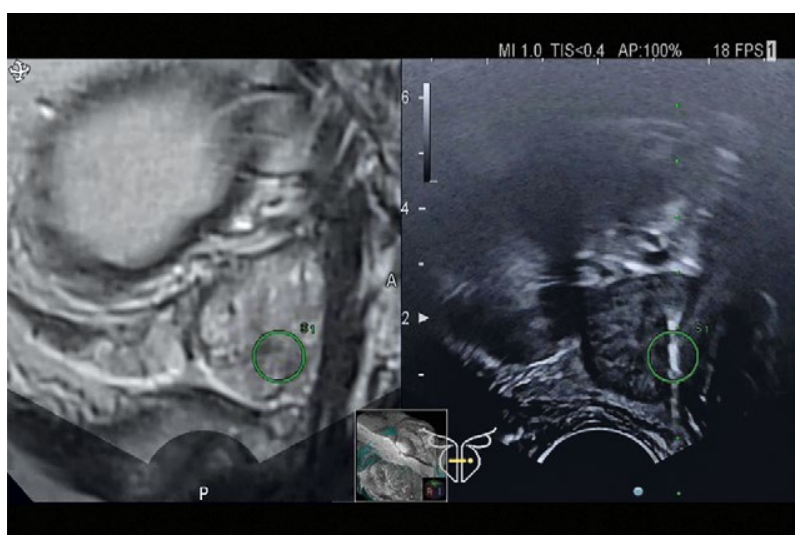

Fig 1. Biopsy of the suspected lesion using the fusion technique. The area identified on MRI as potential malignant is targeted using TRUS during the biopsy

The aim of our study was to compare the cancer detection rate $(\mathrm{CDR})$ and the clinically significant prostate cancer detection rate (csPCa) of the magnetic resonance/ ultrasound fusion Tbs with those of the standard systematic biopsy and those of the two techniques combined. We present our experience with biopsy in naïve patients and in patients with a prior negative biopsy.

\section{Materials and methods}

Our retrospective study included all the patients who underwent MRI fusion biopsy between August 2017 and February 2020 in our centre. All patients signed an informed consent before the procedure. An approval from our local Ethics Committee was obtained prior to starting this study. Indications for performing mMRI were suspicious prostate specific antigen (PSA) levels or abnormal digital rectal examination (DRE). In accordance with our inclusion criteria, we analysed 182 consecutive patients with a PI-RADSv2 score of $\geq 3$. All patients underwent imaging studies in external clinics with experience in multiparametric MRI. In all cases, mMRI was performed with $1.5 \mathrm{~T}$ or $3 \mathrm{~T}$ machines, following the guidelines of the European Society of Urogenital Radiology (multiplanar T2-weighted images, diffusion-weighted imaging, dynamic contrast-enhanced MRI and delayed T1-weighted images with fat suppression). For patients with previous prostate biopsy, mMRI was performed 3 months after the biopsy. For the classification of the lesions, PI-RADSv2 score was used $[8,10]$. For patients with 2 or more lesions, only the lesion with the highest PI-RADSv2 score was analysed.

All biopsies were performed by a transrectal approach under local anaesthesia (5cc of $2 \%$ lidocaine injected between prostate base and seminal vesicle - the region where both neurovascular bundles are found) or

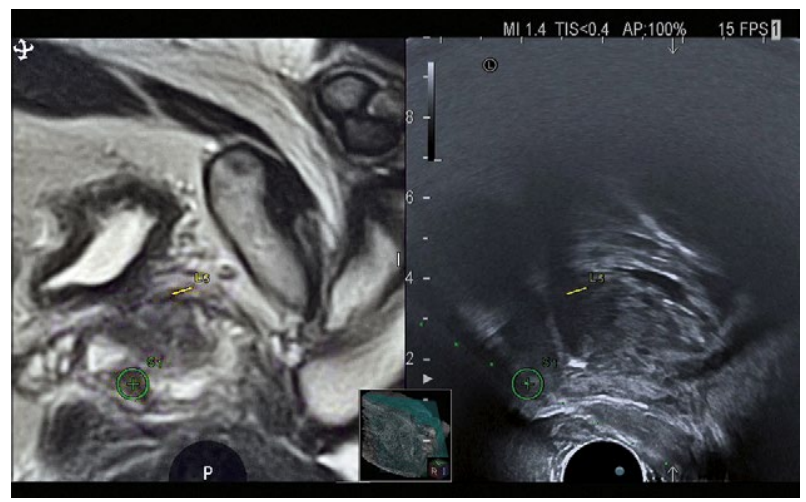

Fig 2. Comparative aspect of MRI and ultrasound of the prostate showing the suspected lesion. The fusion technique allows real time exploration of the areas highlighted as suspicious by the MRI.

intravenous anaesthesia with propofol and midazolam. Antibiotic prophylaxis was done with fluoroquinolones or targeted prophylaxis in patients with fluoroquinolone resistance. The biopsies were performed by the same urologist, with significant experience in the field.

All samples were collected in one session for both Tbs and Sbs by the same urologist, experienced and trained in performing both systematic and targeted biopsies. Biopsies were performed with a Hitachi ultrasound machine, using HI/RVS software for the fusion biopsy. First, the mMRI T2-weighted images on axial and sagittal planes were imported on the ultrasound machine. Both planes were synchronized and the suspected lesions were marked. After the lesion was identified on mMRI images it was fused with the ultrasound image by using anatomical landmarks (ie, urethra, prostate margin, bladder neck). After the images were synchronized, Tbs was performed by collecting 2-4 cores from each suspected lesion. Then Sbs was performed as a 12-core biopsy (fig 1, fig 2). Samples were taken from each lobe with a 22 $\mathrm{mm}$ needle from the lateral and medial part of the basis, middle part and apex. Biopsy cores were sent separately to the pathology department where they were evaluated. According to the International Society of Urological Pathology (ISUP), significant PCa was defined as any Gleason $\geq 3+4 \mathrm{PCa}$ in at least one core (ISUP grade $\geq 2$ ) [11]

\section{Statistical analysis}

Statistics included descriptive variables for different categories and medians and interquartile ranges were reported. We compared detection rates for $\mathrm{PCa}$ and $\mathrm{csPCa}$ between groups. Chi-square test examined the statistical significance. The Mann-Whitney U test examined the statistical significance of medians. We compared CDRs and csPCa detection rates between different biopsy groups (Tbs, Sbs, Tbs + Sbs). Stratification was made depending on previous biopsy status and on the PI-RADSv2 lesion 
score. All tests were two sided, with a level of significance set at $\mathrm{p}<0.05$.

\section{Results}

Out of the 182 patients included in the study, 104 (57.1\%) were biopsy naïve patients and 78 (42.9\%) had previous biopsies. The details about age, median PSA level prior to biopsy, median free-PSA/PSA ratio, median number of PI-RADS and median number of cores taken per patient are detailed in Table I.

The overall detection rate (CDR) was 57.1\% for Tbs alone and $55.5 \%$ for Sbs alone with an increase to $60.4 \%$ for combination of $\mathrm{Tbs}+\mathrm{Sbs}$. Detection rates for significant PCa were $48.4 \%$ for Tbs, $44.5 \%$ for Sbs and $51.1 \%$ for $\mathrm{Tbs}+\mathrm{Sbs}$. In biopsy naïve patients CDR for
Tbs was $67.3 \%$ while in the group with previous biopsy was $43.7 \%$. For Sbs the CDR was $66.4 \%$ in biopsy naive patients and $43.6 \%$ in group with previous negative biopsy (all $\mathrm{p}<0.05$ for biopsy naïve vs. previous biopsy). After stratification, according to the previous biopsy, csPCa detection rates for $\mathrm{Tbs}$, Sbs, and $\mathrm{Tbs}+\mathrm{Sbs}$ were, respectively, $56.7 \%, 52.9 \%$ and $59.6 \%$ for biopsy-naïve patients; $37.2 \%, 33.3 \%$, and $39.7 \%$ for patients with previous negative biopsy (all $\mathrm{p}<0.01$ for biopsy naïve vs previous biopsy) (Table II).

PI-RADSv2 score was 3 in $54(29.7 \%)$ cases, 4 in $96(52.7 \%)$ cases and 5 in $32(17.6 \%)$ cases. By previous biopsy PI-RADSv2 score was 3 in $23.1 \%$ cases, 4 in $53.8 \%$ cases, and 5 in $23.1 \%$ cases for biopsy naïve patients and $38.5 \%, 51.3 \%$ and $10.3 \%$ for patients with previous biopsy.

Table I. Distribution of patients based on previous biopsy status

\begin{tabular}{llll}
\hline & Overall $(\mathbf{n = 1 8 2})$ & No previous biopsy $(n=\mathbf{1 0 4 , 5 7 . 1 \%})$ & Previous biopsies $(\mathbf{n = 7 8 , 4 2 . 9 \% )}$ \\
\hline Age (years) & $62(49-75)$ & $61(49-73)$ & $62(54-75)$ \\
PSA (ng/ml) & $7.4(4.2-14.3)$ & $6.9(4.2-10.8)$ & $9.6(7.2-14.3)$ \\
Prostate volume (ml) & $39(25-81)$ & $36(25-60)$ & $48(38-81)$ \\
MRI lesions) & $1(1-2)$ & $1(1-2)$ & $1(1-2)$ \\
Cores taken & $15(14-17)$ & $15(14-17)$ & $16(15-17)$ \\
Cores taken per lesion & $3(2-5)$ & $3(2-5)$ & $4(3-5)$ \\
Cores taken out of lesion & $12(11-14)$ & $12(11-13)$ & $12(12-14)$ \\
PI-RADS score & & & $32(38.5)$ \\
3 & $54(29.7)$ & $32(23.1)$ & $38(51.3)$ \\
4 & $86(52.7)$ & $48(53.8)$ & $8(10.3)$ \\
5
\end{tabular}

The results are expressed as median (IQR) or number (\%).

Table II. Cancer detection rate and clinically significant prostate cancer detection rate by targeted biopsy, systematic biopsy and a combination of both, based on previous biopsies status

\begin{tabular}{|c|c|c|c|c|}
\hline & Overall $(n=182)$ & No previous biopsy $(n=104,57.1 \%)$ & Previous biopsies $(n=78,42.9 \%)$ & p value \\
\hline Tbs CDR & $104(57.1)$ & $70(67.3)$ & $34(43.7)$ & $<0.01$ \\
\hline Sbs CDR & $101(55.5)$ & $67(64.4)$ & $34(43.6)$ & $<0.01$ \\
\hline Sbs + Tbs CDR & $110(60.4)$ & $71(68.3)$ & $39(50)$ & $<0.05$ \\
\hline Tbs csPCa & $88(48.4)$ & $59(56.7)$ & $29(37.2)$ & $<0.01$ \\
\hline Sbs csPCa & $81(44.5)$ & $55(52.9)$ & $26(33.3)$ & $<0.01$ \\
\hline $\mathrm{Tbs}+\mathrm{Sbs} \operatorname{csPCa}$ & $93(51.1)$ & $62(59.6)$ & $31(39.7)$ & $<0.01$ \\
\hline \multicolumn{5}{|l|}{ ISUP } \\
\hline No cancer & $72(39.6)$ & $33(31.7)$ & $39(50)$ & \\
\hline 1 & $17(9.3)$ & $9(8.7)$ & $8(10.3)$ & \\
\hline 2 & $31(17)$ & $22(21.2)$ & $9(11.5)$ & \\
\hline 3 & $20.9(38)$ & $25(24)$ & $13(16.7)$ & \\
\hline 4 & $13(7.1)$ & $8(7.7)$ & $5(6.4)$ & \\
\hline 5 & $11(6)$ & $7(6.7)$ & $4(5.1)$ & \\
\hline
\end{tabular}

The results are expressed as number $(\%)$. $\mathrm{CDR}=$ Cancer detection rate; $\mathrm{PCa}=$ Prostate cancer; $\mathrm{cs} \mathrm{PCa}=$ clinically significant prostate cancer; Tbs = targeted biopsy; Sbs = systematic biopsy; ISUP = International Society of Urological Pathology 
Table III. Cancer detection rate and clinically significant prostate cancer detection rate by targeted biopsy, systematic biopsy and a combination of both, based on PI-RADS score

\begin{tabular}{llllll}
\hline & $\begin{array}{l}\text { PI-RADS 3 } \\
(\mathbf{n = 5 4 , 2 9 . 7 \% )}\end{array}$ & $\begin{array}{l}\text { PI-RADS 4 } \\
(\mathbf{n = 9 6 , 5 2 . 7 \% )}\end{array}$ & $\begin{array}{l}\text { PI-RADS 5 } \\
(\mathbf{n = 3 2 , 1 7 . 6 \% )}\end{array}$ & $\begin{array}{l}\text { p value } \\
\text { (PI-RADS 3 vs 4) }\end{array}$ & $\begin{array}{l}\text { p value } \\
\text { (PI-RADS 4 vs 5) }\end{array}$ \\
\hline PSA (ng/ml) & $7.1(4.2-10.4)$ & $7.4(6.1-12.1)$ & $8.9(6-14.3)$ & & 0.04 \\
Tbs CDR & $14(25.9)$ & $63(65.6)$ & $27(84.4)$ & $<0.01$ & 0.3 \\
Sbs CDR & $17(31.5)$ & $61(63.5)$ & $23(71.9)$ & $<0.01$ & 0.059 \\
Tbs + Sbs CDR & $19(35.2)$ & $64(66.7)$ & $27(84.4)$ & $<0.01$ & 1.2 \\
Tbs csPCa & $13(24.1)$ & $52(54.2)$ & $23(71.9)$ & $<0.01$ & 0.04 \\
Sbs csPCa & $11(20.4)$ & $47(49)$ & $23(71.9)$ & $<0.01$ & 0.059 \\
Tbs + Sbs csPCa & $15(27.8)$ & $54(56.3)$ & $24(75)$ & $<0.01$ & \\
ISUP in lesion & & & & \\
$\quad$ No PCa & $40(74.1)$ & $33(34.4)$ & $5(15.6)$ & & \\
1 & $1(1.9)$ & $11(11.5)$ & $4(12.5)$ & & \\
2 & $7(13)$ & $21(21.9)$ & $8(25)$ & & \\
3 & $5(9.3)$ & $19(19.8)$ & $6(18.8)$ & & \\
4 & $0(0)$ & $8(8.33)$ & $4(12.5)$ & & \\
5 & $1(1.9)$ & $4(4.2)$ & $5(15.6)$ & & \\
\hline
\end{tabular}

The results are expressed as median (IQR) or number $(\%)$. CDR $=$ Cancer detection rate; PCa = Prostate cancer; csPCa $=$ clinically significant prostate cancer; Tbs = targeted biopsy; Sbs = systematic biopsy; ISUP = International Society of Urological Pathology

The overall CDR stratification by PI-RADSv2 score for Tbs was $25.9 \%, 65.6 \%$ and $84.4 \%$ for score 3,4 and 5 . The CDRs for PI-RADSv2 score 3, 4 and 5 were $31.5 \%$, $63.5 \%$ and $71.9 \%$ for Sbs alone and $35.2 \%, 66.7 \%$ and $84.4 \%$ for Tbs + Sbs, respectively. CDRs of clinically significant PCa for PI-RADSv2 score 3, 4 and 5 were $24.1 \%, 54.2 \%$ and $71.9 \%$ for Tbs alone; $20.4 \%, 49 \%$, $71.9 \%$ for Sbs alone; and $27.8 \%, 56.3 \%$ and $75 \%$ for the combination of Tbs + Sbs (Table III).

In biopsy-naïve patients, CDRs were higher. For Tbs they were $29.2 \%, 76.8 \%$, and $83.3 \%$ for PI-RADS 3, 4, and 5 ; and $29.2 \%, 75 \%$ and $75 \%$ for Sbs alone. In combination Tbs + Sbs CDRs were 33.3\%, 76.8\% and 83.3\%, respectively.

The complication rate was low (1.6\%), including 1 patient with complicated UTI which required IV antibiotics and 2 patients with haematuria.

\section{Discussions}

Magnetic resonance/ultrasound fusion Tbs is widely used in the diagnostic of prostate cancer ( $\mathrm{PCa}$ ) as recent guidelines recommend [8]. Our study complements the previous published results, demonstrating that using the fusion technique to target suspect lesions, followed by systematic biopsies the, detection rates of $\mathrm{PCa}$ and csPCa increases.

$\mathrm{PCa}$ detection rate in our study was superior compared to a study by Zalesky $\mathrm{M}$ et al who reported $38.96 \%$, $27.92 \%$ and $34.42 \%$, for Tbs + Sbs, Tbs and Sbs [12].
Borkowetz A et al also reported lower rates - 52\%, 44\% and 35\% for Tbs + Sbs, Tbs and Sbs. However, Tbs had a higher CDR than Sbs, comparable with our results [3]. We consider our higher detection rates are due to the experience of a single, previously trained urologist, working in a high volume medical facility.

Argun et al reported an overall CDR of $53.2 \%$ with CDR for Sbs of $49 \%$ and for Tbs of $46 \%$ [13]. Our opinion is that higher experience and expertise with targeted biopsy leads eventually to similar or even better CDR than systematic biopsy alone. Maxeiner et al reported higher CDRs of $67 \%, 70 \%$, and $77 \%$ for Tbs, Sbs, and Tbs + Sbs, respectively [2]. The lower CDRs in our cohort might be explained because mMRI was performed in different medical centers with varying generation machines [14-16].

We found no significant difference between CDRs for Tbs alone and Sbs alone. This contrasts with van der Leest et al, and with Pokorny et al [17,18] where Tbs alone had higher rates compared with Sbs alone. We cannot exclude the influence of the mMRI results while performing the systematic biopsy, although in our centre the biopsies were performed in a standardized manner.

Previous studies show lower CDRs for patients with previous negative biopsies [12,19-21]. Our results point to the same conclusion, with CDRs for patients with previous negative biopsy being lower, in line with the results of Kesch et al, who calculated it at 52\% [22]. This correlates with EAU guidelines which recommend mMRI after a negative biopsy and fusion biopsy if necessary [8]. 
When compared the CDRS based on PI-RADSv2 score, we found the higher the score, the higher the detection rate, similar to the PRECISION trial results [23]. In our study, the Sbs improved the csPCa CRDs mostly in PI-RADSv2 score 3 and 5.

Our main limitation is the retrospective type of our data. All biopsies were done in one centre, but mMRI images were obtained from multiple clinics and were performed by many radiologists, although all experienced in mMRI. Our study lacks a control group which could give additional information on the benefit of prebiopsy mMRI. The value of the PI-RADS score might be examinator-dependent. It also would have been of great interest to evaluate the outcome of the patients based on the type of biopsy performed; however, our healthy system's particularities make the follow up difficult once the patient is redirected towards other specialties.

\section{Conclusions}

Magnetic resonance/ultrasound fusion targeted biopsy is now the method of choice for prostate cancer detection. By adding systematic biopsy results to those of the targeted biopsy, the rate of detection for clinically significant prostate cancer increases. Detection rates also increase for higher PI-RADSv2 scores. Our results demonstrate that when Tbs is considered due to a PI-RADS $\geq 3$ lesion on mMRI, combined Tbs + Sbs increases the overall CDR and csPCa detection rates. The impact of our results in medical practice is measured through the 8 cases of PCa that would have been missed by Tbs alone.

\section{Conflict of interest: none}

\section{References}

1. Ahmed HU, El-Shater Bosaily A, Brown LC, et al. Diagnostic accuracy of multi-parametric MRI and TRUS biopsy in prostate cancer (PROMIS): a paired validating confirmatory study. Lancet 2017;389:815-822.

2. Maxeiner A, Kittner B, Blobel C, et al. Primary magnetic resonance imaging/ultrasonography fusion-guided biopsy of the prostate. BJU Int 2018;122:211-218.

3. Borkowetz A, Zastrow S, Platzek I, et al. 209 Comparison of MRI/ultrasound-fusion-biopsy to systematic prostate biopsy in prediction of tumour aggressiveness and final histopathology. Eur Urol Suppl 2015;14:e209.

4. Wegelin O, van Melick HHE, Hooft L, et al. Comparing Three Different Techniques for Magnetic Resonance Imaging-targeted Prostate Biopsies: A Systematic Review of In-bore versus Magnetic Resonance Imaging-transrectal Ultrasound fusion versus Cognitive Registration. Is There a Preferred Technique? Eur Urol 2017;71:517-531.

5. Delongchamps B, Lefevre A, Beuvon F, et al. 955 Multiparametric MRI-TRUS fusion targeted prostate biopsies:
Characteristics of MRI undetected tumour foci. Eur Urol Suppl 2014;13:e955.

6. Fojecki G, Friis J, Carlsson S, Tiessen S. Is MRI/TRUS guided fusion prostate biopsy the way forward? Eur Urol Suppl 2017;16:e2297.

7. Rouvière $\mathrm{O}$, Puech $\mathrm{P}$, Renard-Penna $\mathrm{R}$, et al. Use of prostate systematic and targeted biopsy on the basis of multiparametric MRI in biopsy-naive patients (MRI-FIRST): a prospective, multicentre, paired diagnostic study. Lancet Oncol 2019;20:100-109.

8. Mottet N, Cornford P, van den Bergh RCN, et al. EAU-ESTRO-ESUR-SIOG guidelines on prostate cancer. Presented at the EAU Annual Congress Amsterdam 2020. ISBN 978-94-92671-07-3.

9. Barentsz JO, Weinreb JC, Verma S, et al. Synopsis of the PI-RADS v2 guidelines for multiparametric prostate magnetic resonance imaging and recommendations for use. Eur Urol 2016;69:41-49.

10. Mertan FV, Greer MD, Shih JH, et al. Prospective evaluation of the Prostate Imaging Reporting and Data System Version 2 for prostate cancer detection. J Urol 2016;196:690-696.

11. Epstein JI, Egevad L, Amin MB, et al. The 2014 International Society of Urological Pathology (ISUP) consensus conference on Gleason grading of prostatic carcinoma: definition of grading patterns and proposal for a new grading system. Am J Surg Pathol 2016;40:244-252.

12. Zalesky M, Stejskal J, Ryznarova Z, et al. Detection rate of significant prostate cancer in MRI fusion prostate biopsy. Eur Urol Suppl 2016;15:e1349-e1350.

13. Argun OB, Obek C, Tuna BM, et al. MRI Guided TRUS Fusion Prostate Biopsy. Eur Urol Suppl 2017;16:e2284.

14. Margel D, BerY, Sela S, et al. PT115 - The learning curve of MRIUS fusion prostate biopsies. Eur Urol Suppl. 2018;17:e1885.

15. Westhoff N, Siegel F, Peter C, et al. 618 - Defining the target prior to prostate fusion biopsy: The effect of MRI reporting on cancer detection. Eur Urol Suppl 2018;17:e890.

16. Futterer JJ, Briganti A, De Visschere P, et al. Can clinically significant prostate cancer be detected with multiparametric magnetic resonance imaging? A systematic review of the literature. Eur Urol 2015;68:1045-1053.

17. Sidana A, Watson MJ, George AK, et al. Fusion prostate biopsy outperforms 12-core systematic prostate biopsy in patients with prior negative systematic biopsy: a multiinstitutional analysis. Urol Oncol 2018;36:341.e1-341.e7.

18. van der Leest M, Cornel E, Israël B, et al. Head-to-head comparison of transrectal ultrasound-guided prostate biopsy versus multiparametric prostate resonance imaging with subsequent magnetic resonance-guided biopsy in biopsy-naive men with elevated prostate-specific antigen: a large prospective multicenter clinical study. Eur Urol 2019;75:570-578.

19. Pokorny MR, de Rooij M, Duncan E, et al. Prospective study of diagnostic accuracy comparing prostate cancer detection by transrectal ultrasound-guided biopsy versus magnetic resonance (MR) imaging with subsequent MRguided biopsy in men without previous prostate biopsies. Eur Urol 2014;66:22-29. 
20. Djavan B, Zlotta A, Remzi M, et al. Optimal predictors of prostate cancer on repeat prostate biopsy: a prospective study of 1,051 men. J Urol 2000;163:1144-1148.

21. Aranda-Lozano J, Sierra-Labarta R, de Pablo-Cárdenas Á, Sánchez-Zalabardo D. First prostate biopsy vs rebiopsy: analysis of our series. Arch Esp Urol 2018;71:825-831.
22. Kesch C, Radtke JP, Distler F, et al. Multiparametric MRI and MRI-TRUS fusion-biopsy in patients with prior negative prostate biopsy. Urologe A 2016;55:1071-1077.

23. Kasivisvanathan V, Rannikko AS, Borghi M, et al. MRItargeted or standard biopsy for prostate-cancer diagnosis. N Engl J Med 2018;378:1767-1777. 